\title{
Water Content Continuous Monitoring of Grapevine Xylem Tissue Using a Portable Low-Power Cost-Effective FMCW Radar
}

\author{
Carlos Quemada Mayoral ${ }^{(0}$, Cebrián García González, Juan Carlos Iriarte Galarregui, Diana Marín, \\ Diego Gastón, Carlos Miranda, Ramón Gonzalo, Member, IEEE, Itziar Maestrojuán, \\ Luis Gonzaga Santesteban, and Iñigo Ederra ${ }^{\circledR}$, Member, IEEE
}

\begin{abstract}
This paper presents the real-time monitoring of a grapevine's water content that flows up through the xylem tissue by means of a frequency-modulated continuous-wave radar. The application of an optimization process, based on the superresolution multiple signal classification algorithm, has enabled the reduction of the bandwidth required to discern the xylem water content and, thus, the operating frequency, achieving a depth resolution of at least $3 \mathrm{~mm}$. This design advantage has resulted in a significant step forward toward a real life application, allowing the use of fully-integrated off-the-shelf components in order to implement a completely portable low-power cost-effective radar at $23.1 \mathrm{GHz}$ with a 3.4-GHz bandwidth. The sensor performance has been evaluated by means of three different experiments: irrigation cycles, day/night cycles, and comparison between irrigation cycles at different temperatures. From the experimental results, it is possible to assert that the contactless sensor presented in this paper is very sensitive to changes in the plant's water content, differentiating between daytime and nighttime. In addition, it has been proven that temperature has a noticeable influence over the evapotranspiration, observing negative drying slopes of 5.62 and $6.28 \mathrm{mV} / \mathrm{cycle}$ at $23{ }^{\circ} \mathrm{C}$ and $26{ }^{\circ} \mathrm{C}$, respectively.
\end{abstract}

Index Terms-Contactless sensor, dielectric permittivity, frequency-modulated continuous-wave (FMCW) radar, group velocity, multiple signal classification (MUSIC) algorithm, range resolution, xylem water content.

Manuscript received August 9, 2018; revised November 16, 2018 and February 7, 2019; accepted February 7, 2019. This work was supported in part by the Spanish Ministry of Economy and Competitiveness under Project TEC2016-76997-C3-1-R and in part by the Navarra Government under Project PI025 VITHZ and Project 0011-1365-2016-000084 RAFF. (Corresponding author: Carlos Quemada Mayoral.)

C. Q. Mayoral and C. G. González are with the Department of Electrical, Electronic and Communications Engineering, Public University of Navarre, 31006 Pamplona, Spain (e-mail: carlos.quemada@unavarra.es).

J. C. I. Galarregui, R. Gonzalo, and I. Ederra are with the Department of Electrical and Electronic and Communications Engineering, Public University of Navarre, 31006 Pamplona, Spain, and also with the Institute of Smart Cities, Public University of Navarre, 31006 Pamplona, Spain (e-mail: jcarlos.iriarte@unavarra.es).

D. Marín, C. Miranda, and L. G. Santesteban are with the Department of Agricultural Engineering, Biotechnology and Food, Public University of Navarre, 31006 Pamplona, Spain (e-mail: gonzaga.santesteban@unavarra.es).

D. Gastón and I. Maestrojuán are with Anteral S.L., 31006 Pamplona, Spain (e-mail: imaestrojuan@anteral.com).

Color versions of one or more of the figures in this paper are available online at http://ieeexplore.ieee.org.

Digital Object Identifier 10.1109/TGRS.2019.2900565

\section{INTRODUCTION}

W ATER availability is one of the key factors that determine agricultural production worldwide [1]. Water is essential for transpiration, a process directly coupled with photosynthesis and, therefore, with carbohydrate production [2]. Growers need tools that allow a proper estimation of plant water status in order to adjust irrigation to plant water requirement, to maximize crop water efficiency, and to avoid unnecessary periods of water shortage [3]. On account of this, real-time monitoring of plant water content is an essential issue in the control of plant hydration level.

There are different techniques to monitor plant water status that can be primarily categorized as destructive [4]-[6] and nondestructive [7]-[15] approaches. Although destructive techniques, such as leaf water potential and thermogravimetric measurements, have proved to be highly reliable, they cannot be applied to the same plant for a long-term monitoring. Among the nondestructive measurements, contactless measurements based on ultrasonic or electromagnetic waves (mostly at microwave and infrared frequencies) have been increasingly employed recently. Electromagnetic techniques are applied to several parts of the plant, such as individual leaves [7]-[9], trunk [10], [11], or canopy [12]-[15]. By means of those applied to whole canopies, which consist of radars or radiometers operating at microwave frequencies, it is not possible to determine the water content of an individual plant. Those applied to single leaves, in turn, might face additional positioning problems for in situ continuous monitoring, primarily due to the movement of the leaves. For this reason, more robust parts of the plant, such as trunk or branches, seem to be more appropriate to implant contactless devices for perennial species such as fruit trees and vines. Concerning the contactless measurements on trunk or branches, all references found in the bibliographic compilation use laboratory equipment, making field measurements very complicated.

In this paper, the continuous monitoring of a grapevine's water status by means of reflectivity measurements on the plant's trunk using a portable radar at $23.1 \mathrm{GHz}$ is presented. The reason to choose this operating frequency is that water is highly reflective in this microwave band, what means that radar transceivers in this frequency band can be excellent noncontact sensors of water content in biological tissues. A first approach 
was followed in the experiments performed in 2015 [10] and 2016 [11] based on the continuous-wave monitoring of grapevines by means of general purpose laboratory equipment, in particular a vector network analyzer. Signal postprocessing allowed the spatial discernment and the behavior study of different plant tissues.

In this paper, a step forward toward a real life application is given, since a completely portable low-power radar is proposed. In addition, it makes use of off-the-shelf components, which makes it a cost-effective device. The whole device has been designed by Anteral, Pamplona, Spain, and optimized for vegetative tissues of specific dimensions. This design flow includes the choice of the operating frequency and bandwidth, the hardware implementation with the corresponding choice of the suitable electronic components, and the proper signal processing using high-resolution algorithms if necessary.

This optimization process has enabled the reduction of the bandwidth and, thus, the operating frequency, required to discern the water content that flows up from the root to the leaves through the xylem. This design advantage has resulted in a reduction of the total power consumption and cost of the final device, as well as in the use of fully-integrated off-theshelf components that have allowed the implementation of a completely portable low-power cost-effective radar.

This paper is organized as follows. Section II introduces the most important theoretical aspects related to the detection of water content in vegetative tissues by means of microwave radars. Afterward, the measurement methodology and setup are described in Section III. Section IV presents the most remarkable experimental results obtained during the measurement stage, and finally, Section V concludes this paper.

\section{BACKGROUND}

\section{A. Dielectric Properties of Water Found in Vegetation}

When a dielectric material is placed in the presence of an electric field, its atoms and molecules are polarized, creating electric dipole moments. The capability of a material to resist the applied electric field is given by its dielectric permittivity [16].

The water molecule possesses a permanent electric dipole moment and responds readily to the application of an electromagnetic field. On account of this, pure water in liquid phase has a high dielectric constant compared to other substances found in vegetation [17]. Since the percentage of water in vegetative tissues is significantly high, taking into account both free and bound water, large reflections will be generated, which depend on the water content of different tissue layers. As a consequence, as it is demonstrated in this paper, radar backscattering can constitute a very effective technique to monitor plant water content in real time by means of a nondestructive method.

Water in plants is distributed in the form of free and bound water [5]. Free water is the amount of water directly available for evapotranspiration and the one that flows up through the xylem. Therefore, from an agricultural point of view, this free form is the most relevant, and the one intended to be detected in this paper. On account of this, the estimation of its dielectric properties is a crucial issue in order to reach enough resolution to be able to detect this tissue. In [5], a thorough model to describe the dielectric constant of free water in vegetation tissues, based on the Debye model [18], is presented. Considering that water salinity is negligible at $23.1 \mathrm{GHz}[5]$ and calculating the model at the average working temperature $\left(24.5^{\circ} \mathrm{C}\right)$, the real and imaginary parts of the relative permittivity can be expressed as follows:

$$
\begin{aligned}
\epsilon_{r}^{\prime} & =4.9+\frac{73.46}{1+\left(\frac{f}{19.4}\right)^{2}} \approx-1.54 f+70.85 \\
\epsilon_{r}^{\prime \prime} & =\frac{73.46\left(\frac{f}{19.4}\right)}{1+\left(\frac{f}{19.4}\right)^{2}} \approx-0.27 f+42.37
\end{aligned}
$$

where $f$ is the operating frequency in gigahertz. The bandwidth of the frequency-modulated continuous-wave (FMCW) radar used in this paper is small enough to apply a linear approximation with a tolerable error, as it is presented in the right side of (1) and (2). The absolute value of the relative error at $23.1 \mathrm{GHz}$ when the linear approximation is applied to $\epsilon_{r}^{\prime}$ and $\epsilon_{r}^{\prime \prime}$ is $0.02 \%$ and $0.12 \%$, respectively. At the lower and upper ends of the frequency range, the maximum error of $\epsilon_{r}^{\prime}$ and $\epsilon_{r}^{\prime \prime}$ increases up to $0.4 \%$ and $0.1 \%$ approximately.

As it will be presented in Section II-C, the depth resolution of an FMCW radar depends on the group velocity at which the electromagnetic wave propagates in the medium. It is possible to derive the relation of group velocity to the propagation constant $\beta$ using the following equation [19]:

$$
V_{g}=\left.\left(\frac{1}{2 \pi} \frac{d \beta}{d f}\right)^{-1}\right|_{f=f_{0}}
$$

where $f_{0}$ is the central operating frequency $(23.1 \mathrm{GHz})$. Considering a lossy dielectric medium, the propagation constant can be calculated by means of the following equation [19]:

$$
\beta=\frac{2 \pi f}{c} \sqrt{\frac{\epsilon_{r}^{\prime}}{2}\left(\sqrt{1+\left(\frac{\epsilon_{r}^{\prime \prime}}{\epsilon_{r}^{\prime}}\right)^{2}}+1\right)}
$$

where $c$ is the speed of light in vacuum. Differentiating $\beta$ with respect to $f$ according to (3), it is possible to obtain the following expression for $V_{g}$ as a function of $f, \epsilon_{r}^{\prime}, \epsilon_{r}^{\prime \prime}, d \epsilon_{r}^{\prime} / d f$, $d \epsilon_{r}^{\prime \prime} / d f$, and $|\epsilon|=\left(\epsilon_{r}^{\prime 2}+\epsilon_{r}^{\prime \prime^{2}}\right)^{1 / 2}$. For further details of this derivation, see Appendix

$$
V_{g}=\frac{2 \sqrt{2} c|\epsilon| \sqrt{\epsilon_{r}^{\prime}+|\epsilon|}}{2|\epsilon|^{2}+f\left(\epsilon_{r}^{\prime} \frac{d \epsilon_{r}^{\prime}}{d f}+\epsilon_{r}^{\prime \prime} \frac{d \epsilon_{r}^{\prime \prime}}{d f}\right)+|\epsilon|\left(2 \epsilon_{r}^{\prime}+f \frac{d \epsilon_{r}^{\prime}}{d f}\right)} .
$$

It is important to note that in order to simplify the final expression for $V_{g}$ and taking into account that $\epsilon_{r}^{\prime}$ and $\epsilon_{r}^{\prime \prime}$ possess similar values at the working frequency $(23.1 \mathrm{GHz})$, $\epsilon_{r}^{\prime}$ and $\epsilon_{r}^{\prime \prime}$ can be replaced in (5) by one half of the sum of $\epsilon_{r}^{\prime}$ and $\epsilon_{r}^{\prime \prime}$ after applying the linear approximation given by (1) and (2). In this way, the final expression for $V_{g}$ is given by

$$
V_{g}=\frac{c \sqrt{56.61-0.9} f}{62.25-1.63 f} \text {. }
$$




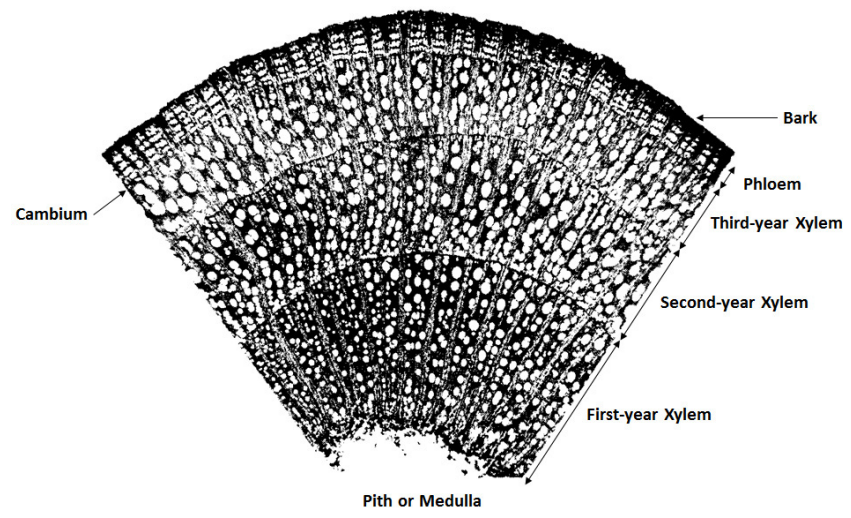

Fig. 1. Transversal section of a three-year-old stem of Vitis vinifera [23].

The group velocity at the central working frequency (23.1 GHz) using (5) is $0.737 \cdot 10^{8} \mathrm{~m} / \mathrm{s}$. The absolute value of the relative error committed at $23.1 \mathrm{GHz}$ when the linear approximation is applied is $0.87 \%$, while at the lower and upper ends of the frequency range, the error increases up to $3.4 \%$ approximately.

\section{B. Wood Structure of the Grapevine Trunk}

Trunks and branches, once formed, do not grow anymore in length, but they just increase their width through the cellular multiplication processes that occur in the vascular cambium. In this vegetative tissue, which is comprised of several thin cell layers, the two major kinds of wood tissues are generated: the phloem, growing outwards, and the xylem, inward [20]. The main role of the phloem is carbohydrate transportation, whereas water movement takes place majorly through the conductive vessels in the xylem [21]. Within the xylem area, the sapwood and the heartwood can be usually distinguished. The sapwood is the truly functional part of the xylem, whereas in the latter, little or no water transport occurs due to vessel clogging with resins or tylose outgrowths as the trunk or branch gets older.

In the case of grapevines, not being truly a tree plant, but a liana, the same structure pattern is observed [22], [23] with average-size conducting xylem vessels [24]. Fig. 1 shows the transversal section of a three-year-old stem of Vitis vinifera. The functional part of the grapevine xylem is mainly constrained to the growth of the last two-three years, due to the great tendency of the plant to generate tylosoids [25], and become totally nonfunctional in six-seven years [26]. Taking into account that the annual trunk growth is between 1.5 and $2.5 \mathrm{~mm}$ in radius for an average vineyard, and that the bark can be easily removed and the phloem as a whole can account for the outer $1 \mathrm{~mm}$ (unpublished data), the thickness of the functional xylem should be between 3 and $5 \mathrm{~mm}$ for an average annual growth of $2 \mathrm{~mm}$ and considering the last two-three years.

\section{FMCW Radar}

The radar used in this paper has been developed by the company Anteral, and it is based on the SiGe $24-\mathrm{GHz}$

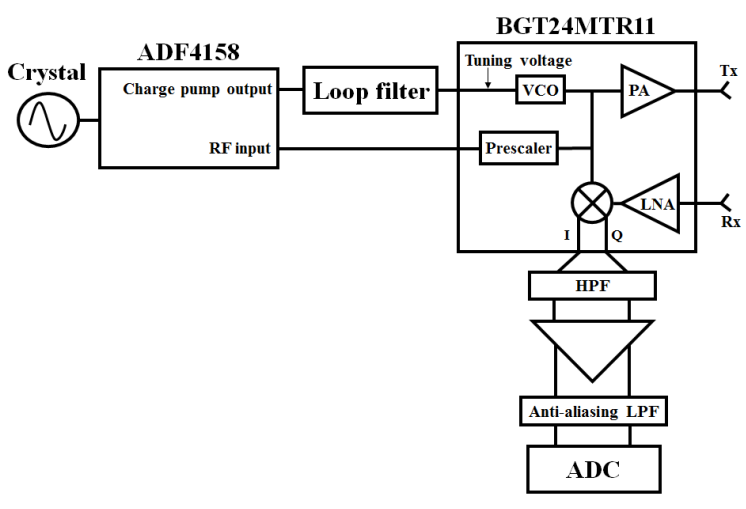

Fig. 2. FMCW radar architecture

transceiver BGT24MTR11 from Infineon Technologies, Munich, Germany, the fractional frequency synthesizer ADF4158 from Analog Devices, Norwood, MA, USA, a low-pass filter for the phase-locked loop (PLL), an analogto-digital converter (ADC) in order to sample the $I$ and $Q$ signals previously amplified, a patch antenna array with an 18-dBi-gain pencil beam pattern, an antialiasing low-pass filter, and a high-pass filter that prevents the transceiver from saturating when objects are placed very close to the antenna. Fig. 2 shows a block diagram of its architecture.

This radar follows an FMCW architecture. In recent decades, the range of applications for this type of radars has increased significantly due to their advantages, such as high resolution and accuracy, lower peak power, and higher operating frequency. In the end, these benefits are reflected in the design of low-power, cost-effective, and miniaturized devices. Obviously, all these performance characteristics mainly depend on the signal bandwidth, working frequency, distance range, voltage-controlled oscillator (VCO) linearity, and VCO phase noise. Among these applications, it is possible to highlight level meters, accurate meters for small displacements and vibrations of particles of different machines and mechanisms, ice thickness measuring systems, water content sensors, concealed weapon and explosives detectors, aircraft landing aid systems, highway traffic monitoring devices, and medical applications [27]-[29].

Different from other techniques, such as the pulse radar, FMCW radar radiates continuous radio frequency (RF) power and changes its operating frequency during the measurement. This frequency modulation is usually generated by means of a frequency synthesizer that offers several modulation ramps. In the case of this paper, a sawtooth waveform has been used for this purpose. FMCW radar divides the transmitted power into two signals. One of them is used as local oscillator (LO) for the receiver mixer, and it is considered the reference signal. The other one, called RF signal, is radiated toward the target, in this case the grapevine, reflected, and sent back to the receiver to be mixed with the reference signal. Due to the delay between both signals at the mixer input, their frequencies at each instant are different and the time delay can be obtained from this frequency difference. Finally, the distance $R$ to the reflecting object can be determined by 
the following equation [28]:

$$
R=\frac{c \Delta f T_{r}}{2 \mathrm{BW}}
$$

where $c$ is the speed of light in vacuum, $\Delta f$ is the frequency difference measured between both signals, $T_{r}$ is the modulation wave period or ramp time, and $\mathrm{BW}$ is the bandwidth of the transmitted signal. If the medium is different from vacuum, the speed of light must be replaced by the group velocity $V_{g}$ calculated by means of (5). The depth resolution $\Delta R$, defined as the minimum separation in depth between two targets of equal cross section that can be resolved as separate targets, can be expressed as follows [28]:

$$
\Delta R=\frac{V_{g}}{2 \mathrm{BW}} .
$$

In the particular case of this paper, the mixer used to implement the receiver is an $I Q$ mixer whose outputs $I$ and $Q$ have a phase difference of $90^{\circ}$ and can be calculated as

$$
\begin{gathered}
I=\frac{A_{\mathrm{Rf}} A_{\mathrm{Lo}}}{2} \cos \left[2 \pi\left(f_{\mathrm{Rf}}-f_{\mathrm{Lo}}\right) t+\varphi_{0}\right] \\
Q=\frac{A_{\mathrm{Rf}} A_{\mathrm{Lo}}}{2} \sin \left[2 \pi\left(f_{\mathrm{Rf}}-f_{\mathrm{Lo}}\right) t+\varphi_{0}\right]
\end{gathered}
$$

where $A_{\mathrm{Rf}}$ and $A_{\mathrm{Lo}}$ are the amplitudes in volts of the mixer input RF and LO signals, respectively, $f_{\mathrm{Rf}}$ and $f_{\mathrm{Lo}}$ are their frequencies, and $\varphi_{0}$ is the phase difference between the initial phases of the RF and LO signals.

In order to obtain the mixer output signal in frequency domain, the most basic signal processing involves calculating the fast Fourier transform (FFT) of the signal $I-j Q$. Since the frequency difference between LO and RF signals is related to the distance $R$ according to (7), it is possible to obtain the aforementioned transform as a function of $R$. Considering the 3.4-GHz bandwidth of the used radar, by means of (8), a depth resolution of $10.83 \mathrm{~mm}$ is obtained. This result has been obtained using a value for the group velocity in the xylem of $0.737 \cdot 10^{8} \mathrm{~m} / \mathrm{s}$, which has been calculated in Section II-A. This resolution could be considered a limiting distance in order to discern different layers of the trunk. Hence, a very wide bandwidth might be needed to satisfy the range resolution requirements in this multilayered structure. Since this option would represent a technological limitation and make the system more expensive, a software solution has been adopted by means of the use of the multiple signal classification (MUSIC) algorithm [30]. This spectral superresolution technique offers higher resolution compared to conventional FFT-based techniques by performing an eigenvalue decomposition of the covariance matrix of the time domain signal. Different from the FFT, the MUSIC algorithm is able to estimate unknown spectral components buried in the noise by using the orthogonality property between the signal and noise subspaces at the expense of computational cost.

\section{MUSIC Algorithm}

This section will briefly describe the fundamentals of the MUSIC algorithm. Considering a multilayered structure, the time domain signal sampled at the ADC output during one modulation period can be expressed as [31]

$$
s(n)=\sum_{k=1}^{K} \alpha_{k} e^{-j 2 \pi f_{k} n T_{s}}+w(n), n=1,2, \ldots, N
$$

where $K$ is the total number of interfaces inside the structure, $\alpha_{k}$ is the reflection coefficient at the $k$ th interface, $f_{k}$ is the beat frequency corresponding to the wave reflected at the $k$ th interface, $T_{\mathrm{S}}$ is the sampling period, $N$ is the total number of samples per modulation period, and $w(n)$ is a Gaussian noise process with zero mean and variance $\sigma^{2} / 2$. Using matrix notation, (11) can be written as

$$
\mathbf{s}=\mathbf{A} \alpha+\mathbf{w}
$$

where $\mathbf{s}=[s(1), s(2), \ldots, s(N)]^{\mathrm{T}}$ is the signal vector, $\boldsymbol{\alpha}=\left[\alpha_{1}, \alpha_{2}, \ldots, \alpha_{K}\right]^{\mathrm{T}}$ is the vector of reflection coefficients, $\mathbf{w}=[w(1), w(2), \ldots, w(N)]^{\mathrm{T}}$ is the noise vector, and the matrix $\mathbf{A}$ is defined as

$$
\mathbf{A}=\left[\begin{array}{cccc}
e^{-j 2 \pi f_{1} T_{\mathrm{s}}} & e^{-j 2 \pi f_{2} T_{\mathrm{s}}} & & e^{-j 2 \pi f_{K} T_{\mathrm{s}}} \\
e^{-j 2 \pi f_{1} 2 T_{\mathrm{s}}} & e^{-j 2 \pi f_{2} 2 T_{\mathrm{s}}} & \cdots & e^{-j 2 \pi f_{K} 2 T_{\mathrm{s}}} \\
\vdots & & \ddots & \vdots \\
e^{-j 2 \pi f_{1} N T_{\mathrm{s}}} & e^{-j 2 \pi f_{2} N T_{\mathrm{s}}} & \cdots & e^{-j 2 \pi f_{K} N T_{\mathrm{s}}}
\end{array}\right]
$$

The covariance matrix of the signal vector $\mathbf{s}$ is calculated as

$$
\Phi=E\left\{\mathbf{s s}^{H}\right\}=\mathbf{A} \boldsymbol{\alpha} \boldsymbol{\alpha}^{H} \mathbf{A}^{H}+\sigma^{2} \mathbf{I}
$$

where $\mathbf{I}$ is the identity matrix and the superscript $\mathrm{H}$ denotes the conjugate transpose of a matrix or vector.

Using the matrix $\Phi$, it is possible to apply the MUSIC algorithm in order to estimate the beat frequencies from the received signal with high resolution.

Therefore, in order to apply the MUSIC algorithm, the first step is to calculate the covariance matrix of $\mathbf{s}$ according to (14). Afterward, it is necessary to apply the eigenvalue decomposition to the covariance matrix. The following equation is obtained:

$$
\boldsymbol{\Phi V}=\mathbf{V}\left[\begin{array}{ccc}
\lambda_{1} & \cdots & 0 \\
\vdots & \ddots & \vdots \\
0 & \cdots & \lambda_{N}
\end{array}\right]
$$

where $\lambda_{i}$ is the $i$ th eigenvalue of $\boldsymbol{\Phi}$ such that $\lambda_{1} \leq$ $\lambda_{2} \leq \ldots \leq \lambda_{N}$ and $\mathbf{V}$ is the matrix whose columns are the corresponding eigenvectors.

The noise subspace is composed of the vectors corresponding to the first $N-K$ columns of $\mathbf{V}$, which are arranged in a new matrix $\mathbf{V}_{\boldsymbol{w}}$, and the $K$ remaining columns constitute the signal subspace. Now, it is possible to calculate the MUSIC frequency spectrum as

$$
S_{\text {MUSIC }}\left(f_{k}\right)=\frac{1}{\boldsymbol{a}_{\mathbf{s}}\left(f_{k}\right) \mathbf{V}_{\boldsymbol{w}} \mathbf{V}_{\boldsymbol{w}}^{\boldsymbol{H}} \boldsymbol{a}_{\mathbf{s}}^{\boldsymbol{H}}\left(f_{k}\right)}
$$

where $\boldsymbol{a}_{\mathbf{s}}\left(f_{k}\right)=\left[e^{-j 2 \pi f_{K} T_{\mathrm{s}}}, e^{-j 2 \pi f_{K} 2 T_{\mathrm{s}}}, \ldots, e^{-j 2 \pi f_{K} N T_{\mathrm{s}}}\right]^{\mathrm{T}}$ is the frequency scanning vector that corresponds to the columns of $\mathbf{A}$. 
In the literature, it has been heuristically demonstrated that MUSIC algorithm offers higher resolution than conventional FFT-based techniques. However, as this resolution improvement depends on multiple parameters such as the available bandwidth, the signal-to-noise ratio, the relative phase difference between different scatterers, and the number of data samples, it is not possible to accurately quantify its value [32]. Because of this, an experimental comparison between both techniques will be presented in Section III-B.

\section{Measurement Methodology}

\section{A. Measurement Setup}

In order to assure accuracy and reliability in the control of external conditions, mainly temperature and lighting, the whole setup, which includes the grapevine and the measurement system, has been settled inside a growth chamber. It is important to note that plant water content depends considerably on these conditions and, therefore, they should be able to be changed at will in order to reproduce different outdoor situations in a short period of time. Different sensors and mechanical devices are in charge of monitoring lighting conditions, temperature, and humidity and can be precisely adjusted to achieve specific plant behaviors. Humidity measurement is based on the soil volumetric water content (VWC) monitoring by means of the capacitance soil moisture probe EC10 from Decagon Devices Inc., Pullman, WA, USA, inserted in the pot. This sensor estimates soil volumetric moisture by determining the apparent permittivity of the soil.

Among different grapevines available for experimental purpose in the greenhouse, a very smooth-trunk plant without any nodes has been chosen in order to avoid the scattering of the reflected signal in multiple directions and penetrate a specific area of the plant that possesses xylem vessels and, consequently, high water content. At the beginning of the experiment, the plant was 14 years old and had been transplanted from the field into a pot three years before the experiment started, sufficient time for the plant to acclimate to its new habitat.

The measurement system consists of an FMCW radar at 23.1 GHz with a 3.4-GHz bandwidth, an ADC to sample the $I$ and $Q$ signals at the mixer output, a data logger with temperature, humidity, and light sensors that records data periodically, and a PC that controls all the previous devices. Fig. 3 shows an illustrative diagram of the measurement setup.

Both the entire FMCW radar and the software application have been optimized in order to improve its sensitivity to water content of vegetative tissues with a structure similar to the grapevine morphology. The hardware part is based on the transceiver BGT24MTR11 from Infineon Technologies, a silicon germanium monolithic microwave integrated circuit (MMIC) for signal generation, and reception operating from 24 up to $26 \mathrm{GHz}$. The VCO and two switchable frequency prescalers are included in the MMIC and have been used to accurately tune the transmitter output frequency by means of the frequency synthesizer ADF4158 from Analog Devices. Among different modulation ramps offered by this PLL, a sawtooth waveform with a ramp time of $5 \mathrm{~ms}$

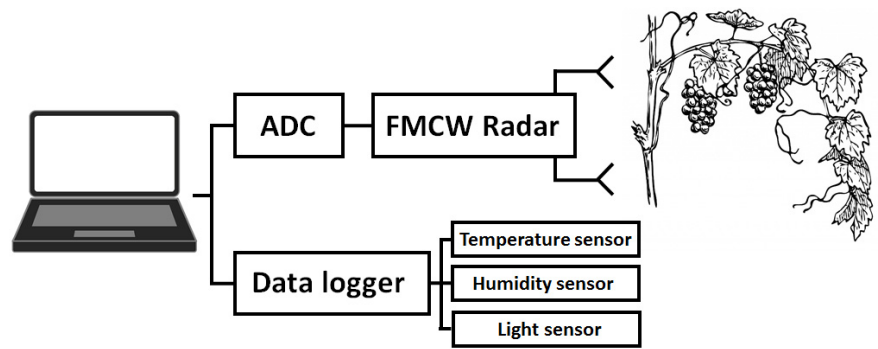

Fig. 3. Measurement setup for monitoring the grapevine water status by means of an FMCW radar. It is comprised by an ADC to sample the data at the mixer output, a data logger with several sensors to record environmental data periodically, and a PC to control all the previous devices.

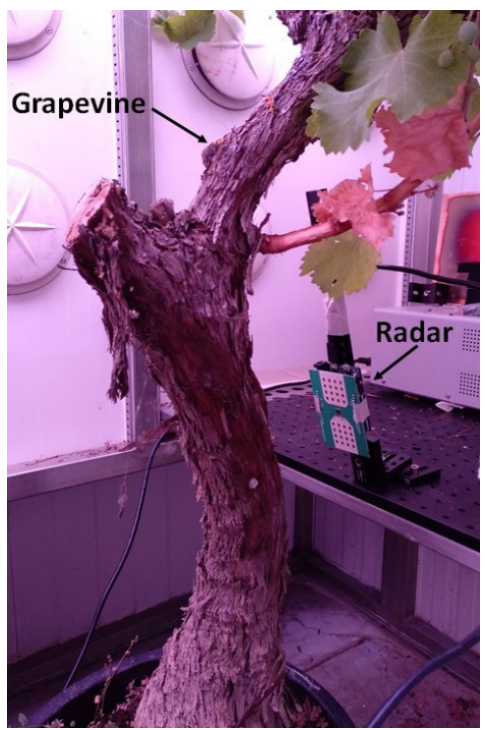

Fig. 4. Photograph of the FMCW radar used to monitor the grapevine.

has been selected. In order to cover the whole voltage range of the ADC and reduce the conversion error, and remove the dc offset of the mixer output signals $I$ and $Q$, the combination of a high-pass filter and an amplifier has been chosen. Depending on the distance range to measure, the high-pass filter attenuates a specific frequency range according to its cutoff frequency, which corresponds to a specific distance range. In this experiment, the radar is placed $10 \mathrm{~cm}$ away from the trunk. Afterward, the amplifier gain is selected in order to reach the maximum voltage range of the ADC. Both the high-pass filter and the amplifier gain have been selected according to the distance at which the grapevine is positioned and its dimensions. Finally, the two signals $I$ and $Q$ have been sampled by means of analog sampling provided by the open-source prototyping platform Arduino Genuino Zero that includes a 12-bit ADC. In Fig. 4, a photograph of the FMCW radar-based measurement setup is presented. The total power consumption and size of the FMCW radar are $170 \mathrm{~mA}$ at $5 \mathrm{~V}$ and $85 \mathrm{~mm} \times 60 \mathrm{~mm} \times 11 \mathrm{~mm}$, respectively.

Another important aspect related to radar signal processing is the calibration technique used to reduce the influence of drifts on the measurements. Two types of calibration have been experimented in this paper: the first one is based on open-air 
measurements without any target and the second one involves measuring the reflection of a piece of absorbent material before every grapevine measurement. Although both methods have been proved satisfactorily, the first type of calibration has been selected for this paper because of its simplicity and accuracy.

\section{B. Bandwidth Optimization}

The manufacturing process of the FMCW radar includes a design flow that optimizes the water detection process of vegetative tissues depending on the specific dimensions of the tissue under study. This design flow includes the choice of the operating frequency and bandwidth, the hardware implementation with the corresponding choice of the electronic components, and the proper signal processing using highresolution algorithms if necessary.

Since the objective of this paper is to detect the water content of vegetative tissues by means of a low-power and cost-effective device, the first step of the design flow is particularly important. On account of this, it is necessary to estimate the resolution improvement after applying the MUSIC algorithm in order to not oversize the final design. This resolution enhancement has been calculated from the experimental results obtained in this paper.

As commented in Section II-C, considering a bandwidth of $3.4 \mathrm{GHz}$, a depth resolution of $10.83 \mathrm{~mm}$ is obtained, which is a limiting distance in order to discern different layers of the trunk, especially the xylem that possesses a width of at least $3 \mathrm{~mm}$ (Section II-B). In order to satisfy the range resolution requirements in this multilayered structure, instead of raising the bandwidth, the MUSIC algorithm has been adopted trying to reduce the cost of the final system. The $I$ and $Q$ signals at the mixer output are sampled at $200 \mathrm{kHz}$ every $2 \mathrm{~min}$. This means that a MUSIC spectrum is calculated every time both signals are sampled.

In order to show the improvement achieved by means of the MUSIC algorithm, a comparison between the FFT and MUSIC spectral techniques has been performed after applying the radar to the same grapevine under study whose experimental results will be presented in Section IV. Since the sampling frequency of the time domain signal is $200 \mathrm{kHz}$ and the ramp time is $5 \mathrm{~ms}$, the number of samples per ramp is 1000. In addition, from all waves reflected on the multilayered structure, a number of $K=40$ have been selected to compute the MUSIC algorithm.

Fig. 5 shows the comparison of the FFT and MUSIC spectra for a specific sampling of the $I$ and $Q$ signals (belonging to the 36th six-hour cycle of the experimental results shown in Section IV) as a function of the distance evaluated from the radar position. Since the trunk is placed at $10 \mathrm{~cm}$, the total distance range has been limited to $1 \mathrm{~m}$. Observing Fig. 5, it is clear that MUSIC spectrum possesses sharper spectral components that the ones of the FFT spectrum, improving the resolution and allowing the discernment of different trunk layers. Another important conclusion drawn from this comparison is that MUSIC algorithm ignores most of the false components considered in the FFT spectrum, which are mainly due to the sidelobes of the main reflections. For the particular case of this

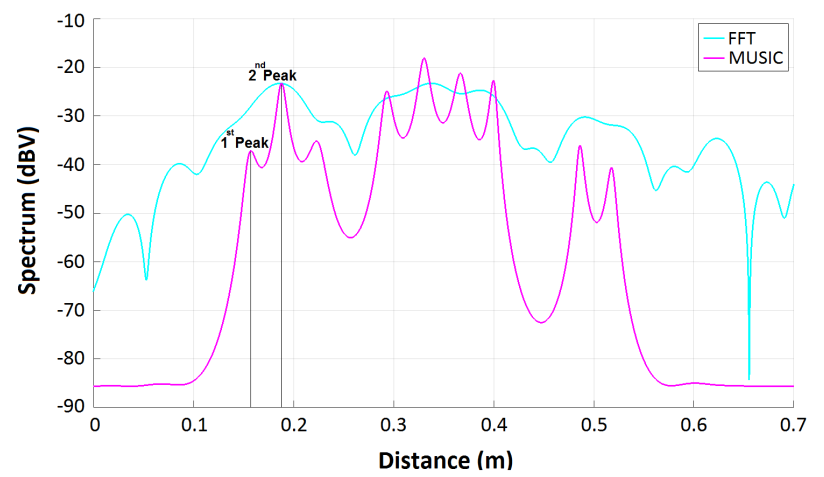

Fig. 5. Comparison between the FFT and MUSIC spectra used to calculate the resolution improvement at $18.8 \mathrm{~cm}$ by using the MUSIC algorithm. The first peak at $15.7 \mathrm{~cm}$ corresponds to the first interface between the air and the trunk, and the second peak at $18.8 \mathrm{~cm}$ corresponds to the xylem tissue.

paper, the spectral component that corresponds to the xylem tissue is the second one, positioned at approximately $18.8 \mathrm{~cm}$ from the radar position. All results presented in Section IV are based on variations of the maximum value of this spectral component. Among the rest of the components, it is worth highlighting the first component placed at approximately $15.7 \mathrm{~cm}$, which corresponds to the first interface between the air and the trunk. This distance does not coincide with the real distance due to the nonlinearity of the VCO. Regarding the position of the second component, it is necessary to add that the dielectric constant of the vegetative tissue positioned between the air and the xylem is different from the one to the air. On account of this, it would be necessary to use the group velocity of the electromagnetic field in this zone in order to calculate the real distance. In order to obtain a reliable value for the permittivity in this zone as a function of the frequency and, thus, a reliable value for the group velocity, it would be necessary to section the trunk, measure the permittivity, and create an empirical model as a function of the frequency. Since this study would go beyond the objective of this paper, which is to detect the xylem tissue rather than calculating its positioning, and the phloem transports free water, it is possible to estimate the group velocity by using the value $0.737 \cdot 10^{8} \mathrm{~m} / \mathrm{s}$ calculated in Section II-A. This results in an approximated real distance of $0.76 \mathrm{~cm}$ between both peaks.

Comparing the full-width at half-maximum of the spectral component that corresponds to the xylem tissue (positioned at approximately $18.8 \mathrm{~cm}$ ) for both spectra in Fig. 5, an approximately five times resolution improvement is achieved. Taking into account that the part of the xylem through which the water flows is at least about $3 \mathrm{~mm}$ thick, as it has been presented in Section II-B, a resolution of $15 \mathrm{~mm}$ (five times higher) would be enough to detect this part on the condition that the MUSIC algorithm is applied afterward. Using now (8), it is possible to estimate that a bandwidth of $2.45 \mathrm{GHz}$ is required to fulfill this resolution requirement. Applying a safety margin of about $1 \mathrm{GHz}$, a bandwidth of $3.4 \mathrm{GHz}$ and an output frequency of $23.1 \mathrm{GHz}$ have been chosen.

Another aspect in Fig. 5 that is worth discussing is the difference between the depth resolution value of $10.83 \mathrm{~mm}$ 


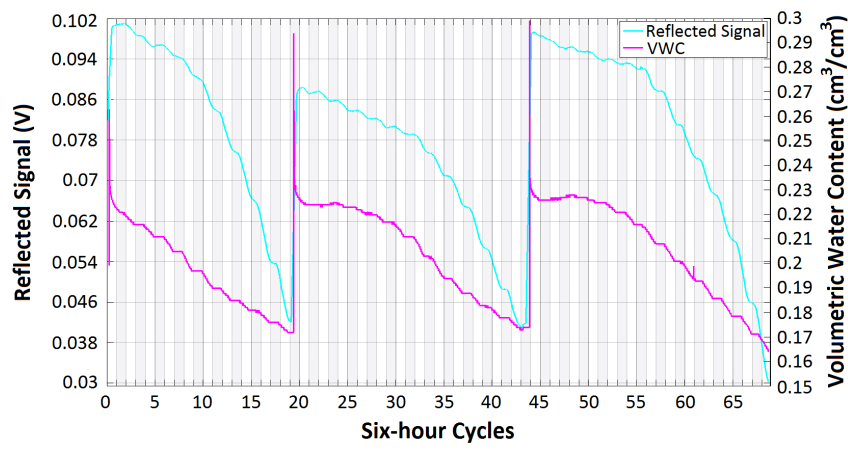

Fig. 6. Irrigation cycles conducted during the experiment. The grapevine was submitted to three irrigation cycles during 18 days with day/night cycles of $6 \mathrm{~h}$ (12-h days). This figure shows the amplitude of the signal reflected by the xylem tissue and the soil VWC as a function of the time.

calculated in Section II-C and the $3-\mathrm{dB}$ peakwidth (44 mm) of the second peak in Fig. 5 for the FFT plot. First of all, it is necessary to clarify that the FFT plot in Fig. 5 has been calculated using the speed of light in vacuum. In order to obtain the 3-dB peakwidth of the second peak, the group velocity in this zone should be used. Consider that the whole peak is due to the xylem tissue and, thus, using a group velocity of $0.737 \cdot 10^{8} \mathrm{~m} / \mathrm{s}$, an approximated real value of $10.81 \mathrm{~mm}$ is obtained. In addition to this, some other factors could affect this difference. As a matter of fact, depth resolution, given by (8), is a theoretical calculation that does not consider important real factors, as the VCO phase noise and the scattering on the plant's trunk, which significantly affect the final result [27].

\section{RESULTS}

With the objective of proving that this portable low-power contactless sensor is able to estimate the plant's water status, three different experiments have been performed: irrigation cycles, day/night cycles, and comparison between irrigation cycles at different temperatures.

\section{A. Irrigation Cycles}

This experiment started on November 2, 2017. The grapevine was submitted to irrigation and drying periods during 18 days with day/night cycles of $6 \mathrm{~h}$. Therefore, the duration of a day in the growth chamber was forced to be $12 \mathrm{~h}$ in order to obtain more information in a shorter time period. Since the grapevine is a multilayered dielectric structure, different peaks will appear in every spectrum due to different reflections that take place in the interfaces between layers where a change of permittivity occurs. Among all these maximum values, the one referred to the xylem tissue, through which the water flows, has been identified by choosing the highest peak of every spectrum at distances shorter than the separation between the trunk and the radar plus the trunk diameter, which has been previously measured. This peak corresponds to the second one, as shown in Fig. 5.

Fig. 6 shows the amplitude of the signal reflected by the xylem tissue and the soil VWC as a function of the time.

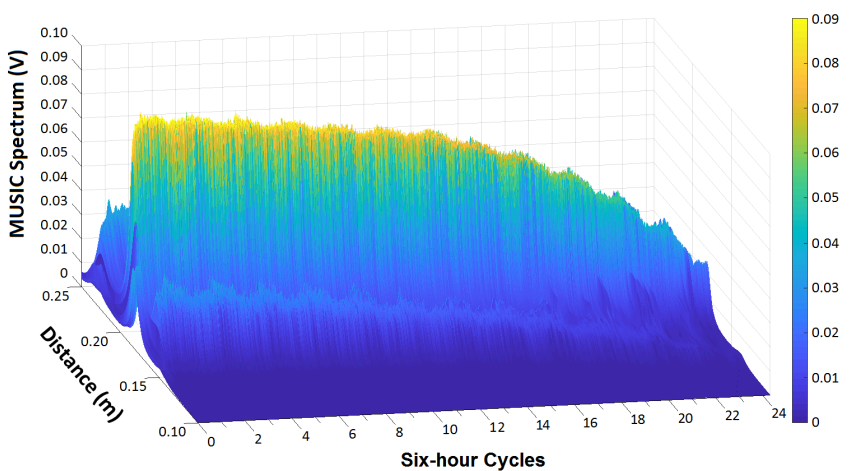

Fig. 7. 3-D MUSIC spectrum for the second irrigation cycle in Fig. 6 as a function of the distance between the radar and the trunk, and the time in six-hour cycles. The maximum values at each instant of time draw the reflected signal for the second irrigation cycle in Fig. 6.

Considering 12-h days, the irrigation days were: the 1 st (the first six-hour cycle), the 10th (the 20th six-hour cycle), and the 22nd (the 44th six-hour cycle) day of the experiment. The dark areas in Fig. 6 correspond with the night periods. The plot for the soil VWC, a surrogate variable for plant water status, has been obtained from the data provided by the humidity probe and for the reflected signal by plotting the maximum values of the second peak of the signal's spectrum at the mixer output calculated by using the MUSIC algorithm and a moving average over 100 samples. The sharp peaks observed for a few minutes at each irrigation event correspond to the irrigation itself when the water content around the soil moisture probe increases, taking some time to return to a state of equilibrium between the soil and the plant's water content.

In order to better understand how Fig. 6 has been obtained, Fig. 7 shows the 3-D MUSIC spectrum for the second irrigation cycle depicted in Fig. 6 as a function of the distance between the radar and the trunk and the time in minutes. The highest spectral component, placed at approximately $18.8 \mathrm{~cm}$, corresponds to the xylem tissue. It can be seen that the maximum values of each MUSIC spectrum draw the second irrigation depicted by the reflected signal in Fig. 6. Finally, another aspect to be noted is that the MUSIC spectrum in Fig. 5 is obtained by means of the intersection of the 3-D MUSIC spectrum in Fig. 7 and the plane 17.26 six-hour cycles.

Three irrigation events can be distinguished in Fig. 6. The amount of water used in the first one was $4 \mathrm{~L}$, whereas in the second and third ones, the amount of water was $8 \mathrm{~L}$. This difference is due to the initial hydration level of the grapevine before the first irrigation, higher than the one before the other two irrigation events. This explains why the initial reflected signal at the first irrigation event increases up to a higher level than the one of the other two irrigations, despite the smaller amount of water used for watering. According to the results shown in Fig. 6, it is possible to assert that the contactless sensor presented in this paper is very sensitive to changes in plant water content. The reflected signal plot follows the trend of VWC closely, even in the instants when a change from day to night happens. If the three drying periods are compared, it is possible to realize that after the first irrigation the plant follows 


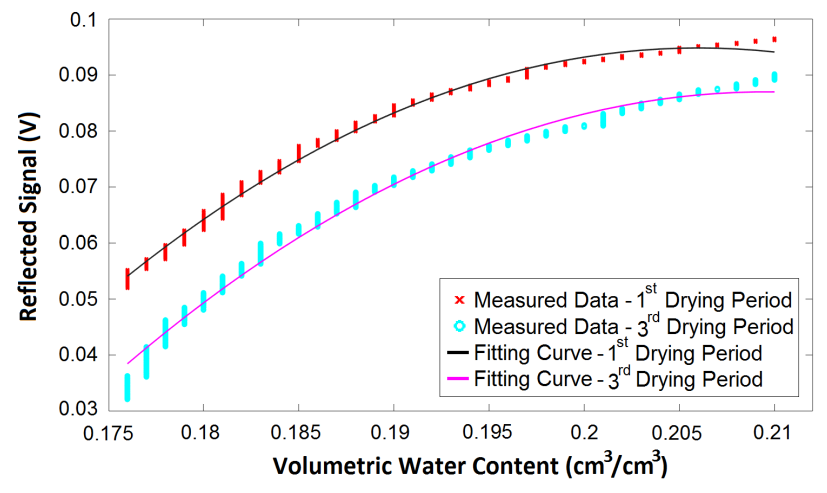

Fig. 8. Second-order curve fitting between the VWC and the reflected signal for the first and third drying periods. For the first one, the relationship between both magnitudes is given by the curve $y=-45.2625 \mathrm{x}^{2}+18.649 \mathrm{x}-1.8261$ with a coefficient of determination $R^{2}=0.9954$. For the third one, the fitting curve $y=-43.0654 \mathrm{x}^{2}+18.0513 \mathrm{x}-1.8046$ is obtained with a coefficient of determination $R^{2}=0.9893$.

a trend slightly different from the one after the other two irrigation cycles. At the beginning of the first drying period, the reflected signal decreases more abruptly than in the other two ones. This might be due to the hydration level of the plant when the watering occurs $(0.2)$. Since at the beginning of the second and the third drying period the hydration level is relatively lower $(0.172)$, the plant experiences a period of acclimatization and needs to retain water before increasing the transpiration rate. Finally, another important aspect to consider is that the reflected signal increases at the time of watering a little sooner than the VWC (100 min). The reason why this occurs is that a moving average over 100 samples has been applied in order to smooth the reflected signal plot.

With the objective of quantifying the degree of similarity between the reflected signal and the VWC, both plots have been related by means of the second order curve fitting shown in Fig. 8. This fitting has been performed for the first and third drying periods. For the first one, the relationship between both magnitudes is given by the curve $y=-45.2625 \mathrm{x}^{2}+18.649 \mathrm{x}-$ 1.8261 with a coefficient of determination $R^{2}=0.9954$. For the third one, the fitting curve $y=-43.0654 x^{2}+18.0513 x-$ 1.8046 is obtained with a coefficient of determination $R^{2}=0.9893$. Although both curves have the same trend, different factors, such as the water status of the plant before watering, the amount of water used for each irrigation, and the time of year, could cause a difference between the mean values of both plots. It is important to mention that the water status of the plant and the amount of water used for watering are different for the first and third irrigation cycles.

\section{B. Day/Night Cycles}

In order to explain the grapevine's behavior during the day and night periods, a zoomed-in view of the graphs in Fig. 6 between the 40th and 44th six-hour cycles is presented in Fig. 9. Since the observation time period is smaller and a greater similarity between both plots is intended to show, a moving average of ten samples has been used. The first thing to be highlighted is that the trend followed by both plots is very similar. As in Section IV-A, the degree of similarity

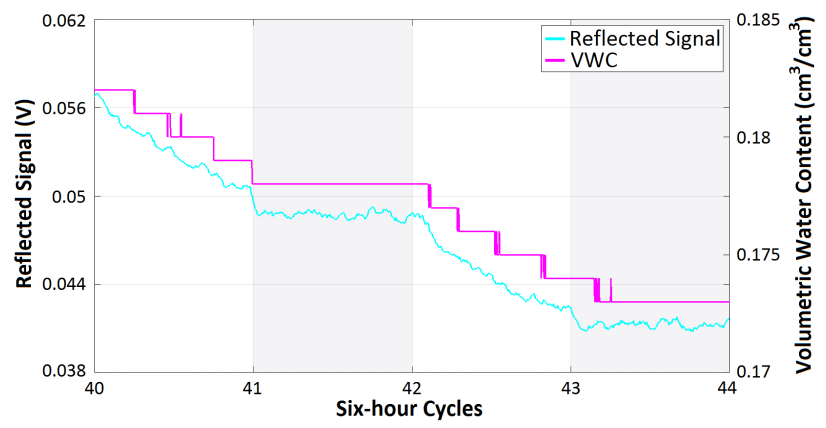

Fig. 9. Zoomed-in view of the graphs in Fig. 6 between the 40th and 44th six-hour cycles.

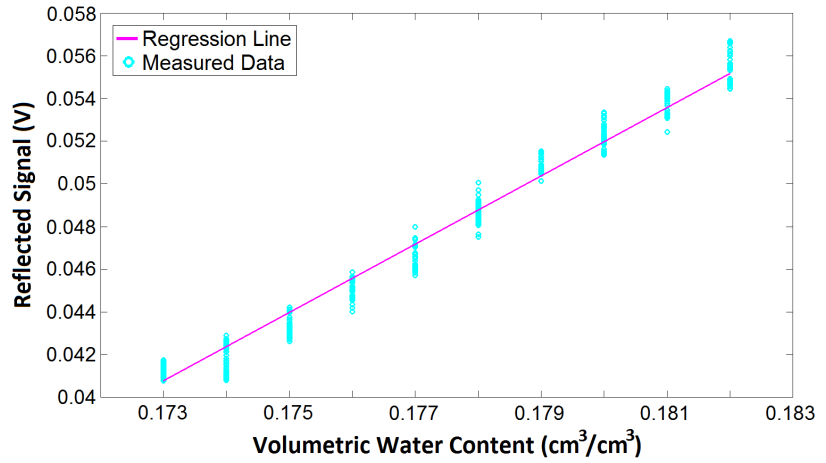

Fig. 10. Linear regression analysis between the VWC and the reflected signal. The relationship between both magnitudes is given by the straight line $y=1.5982 x-0.2357$ with a coefficient of determination $R^{2}=0.9834$.

between both plots has been quantified by means of the linear regression shown in Fig. 10. The relationship between both magnitudes is given by the straight line $y=1.5982 x-0.2357$ with a coefficient of determination $R^{2}=0.9834$.

In addition to this, if a detailed inspection is performed at the plots in Fig. 9, it is easy to realize that the reflected signal value decreases during the day and keeps constant during the night. This phenomenon may be explained by the evapotranspiration process of the grapevine. At dawn, as the presence of light stimulates, photosynthesis stomata are opened and the plant loses water content by transpiration. Since water possesses a high relative permittivity, the reflected signal power decreases during the day. On account of this, new water is uptake through the roots in order to replace the lost quantity. This can be seen in Fig. 9 where both plots, the VWC and the reflected signal, decrease at the same time between the 40th and 41st and 42nd and 43rd six-hour cycles. On the other hand, during the night, due to the absence of light, stomata are closed and the plant keeps the amount of water that had at the end of the day.

\section{Comparison Between Drying Cycles at Different Temperatures}

In order to prove the influence of temperature on plant transpiration, a third experiment based on the comparison between drying periods at different temperatures has been performed. The results of this experiment are presented in Fig. 11, 


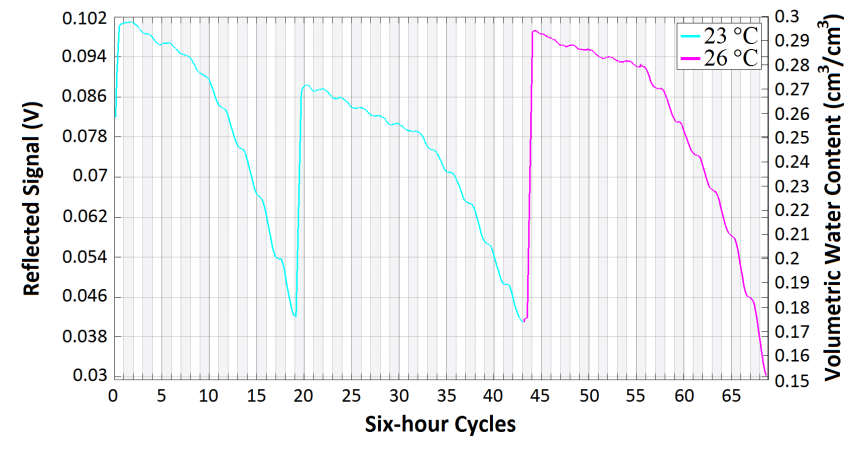

Fig. 11. Drying cycles at different temperatures. During the first two periods, the temperature of the growth chamber was set at $23{ }^{\circ} \mathrm{C}$, whereas during the third period, the temperature was increased to $26^{\circ} \mathrm{C}$. It is the same plot as the one of Fig. 6 for the reflected signal with a different color for the third irrigation cycle.

in which the reflected signal is plotted for three irrigation cycles at two different temperatures. During the first two drying periods, the grapevine was submitted at a temperature of $23{ }^{\circ} \mathrm{C}$, whereas during the third period, the temperature was increased to $26{ }^{\circ} \mathrm{C}$. For a fair comparison, it would be necessary to compare both drying cycles after the period of acclimatization by choosing the most linear zone of the plots. Taking this into account, only the last 6 six-hour cycles of each period have been considered. For the drying period at $23{ }^{\circ} \mathrm{C}$, a negative slope of $5.62 \mathrm{mV} /$ cycle is obtained, whereas at $26{ }^{\circ} \mathrm{C}$, the reflected signal decreases with a slope of $6.28 \mathrm{mV} /$ cycle. From this result, it is possible to assert that temperature has a noticeable influence over the evapotranspiration and it seems clear that the higher the temperature, the higher the evapotranspiration will be.

\section{CONClusion}

Different from other approaches, which use general purpose laboratory equipment making field measurements very complicated, this paper presents a nondestructive low-power cost-effective portable water content monitoring system for vegetative tissues at $23.1 \mathrm{GHz}$. This contactless sensor, based on an FMCW radar, has been developed and optimized for monitoring vegetative tissues of specific dimensions. For the particular case of this paper, since a bandwidth of $3.4 \mathrm{GHz}$ does not provide enough resolution, the MUSIC algorithm has been used. Once this sensor was implemented, three types of measurements were performed: irrigation cycles, day/night cycles, and comparison between irrigation cycles at different temperatures. From these measurements, it is possible to assert that the contactless sensor presented in this paper is very sensitive to changes in plant water content following the trend of VWC closely. In addition, this monitoring system is able to detect plant evapotranspiration differentiating between daytime and nighttime. Finally, it has been proven that temperature has a noticeable influence over the evapotranspiration, coming to the conclusion that the higher the temperature, the higher the evapotranspiration will be.

In addition to this application for water content detection, the potential use of this technology in other research areas might be unique and competitive. The contactless sensor proposed in this paper could be used to control the drought stress in plants, as well as to detect crop diseases that cause dehydration of vegetative tissues inside the trunk. Another important application could be the monitoring of the sap flow velocity passing through the xylem, closely related to the transpiration rate and, thus, to the vapor pressure deficit.

\section{APPENDIX}

This appendix describes the derivation of (5). To start with, the propagation constant $\beta$ can be expressed as follows:

$$
\beta=\frac{\sqrt{2} \pi}{c} \sqrt{f^{2} A}
$$

where $A$ is given by

$$
A=\sqrt{\epsilon_{r}{ }^{2}+\epsilon_{r}^{\prime \prime 2}}+\epsilon_{r}^{\prime} .
$$

In order to better understand the total derivation process, the derivative of $f^{2} A$ with respect to $f$ is calculated

$$
\frac{d\left(f^{2} A\right)}{d f}=2 f\left(|\epsilon|+\epsilon_{r}^{\prime}\right)+f^{2}\left(\frac{d \epsilon_{r}^{\prime}}{d f}+\frac{\epsilon_{r}^{\prime} \frac{d \epsilon_{r}^{\prime}}{d f}+\epsilon_{r}^{\prime \prime} \frac{d \epsilon_{r}^{\prime \prime}}{d f}}{|\epsilon|}\right) .
$$

Applying the definition of group velocity given by (3), the following equation is obtained:

$$
V_{g}=\frac{2 \sqrt{2} c \sqrt{f^{2} A}}{\frac{d\left(f^{2} A\right)}{d f}} .
$$

Replacing in (20), the derivative of $f^{2} A$ with respect to $f$ by the expression given by (19), choosing $|\epsilon|$ as common denominator in (19) and rearranging terms, it is possible to obtain the expression for $V_{g}$ given by (5).

\section{REFERENCES}

[1] P. Steduto, T. C. Hsiao, E. Fereres, and D. Raes, "Crop yield response to water," FAO, Rome, Italy, Irrigation Drainage Paper 66, 2012, pp. $1-500$.

[2] Z. Siddique, S. Jan, S. Imadi, A. Gul, and P. Ahmad, "Drought stress and photosynthesis in plants," in Water Stress and Crop Plants: A Sustainable Approach. Hoboken, NJ, USA: Wiley, 2016.

[3] K. Steppe, D. J. W. De Pauw, and R. Lemeur, "A step towards new irrigation scheduling strategies using plant-based measurements and mathematical modelling," Irrigation Sci., vol. 26, pp. 505-517, Sep. 2008

[4] H. G. Jones, "Monitoring plant and soil water status: Established and novel methods revisited and their relevance to studies of drought tolerance," J. Exp. Botany, vol. 58, no. 2, pp. 119-130, Jan. 2007.

[5] K. C. McDonald, R. Zimmermann, and J. S. Kimball, "Diurnal and spatial variation of xylem dielectric constant in Norway Spruce (Picea abies [L.] Karst.) as related to microclimate, xylem sap flow, and xylem chemistry," IEEE Trans. Geosci. Remote Sens., vol. 40, no. 9, pp. 2063-2082, Sep. 2002.

[6] K. C. McDonald, R. Zimmermann, J. Way, and W. Chun, "Automated instrumentation for continuous monitoring of the dielectric properties of woody vegetation: System design, implementation, and selected in situ measurements," IEEE Trans. Geosci. Remote Sens., vol. 37, no. 4, pp. 1880-1894, Jul. 1999.

[7] A. Afsharinejad, A. Davy, and M. Naftaly, "Variability of terahertz transmission measured in live plant leaves," IEEE Geosci. Remote Sens. Lett., vol. 14, no. 5, pp. 636-638, May 2017. 
[8] E. Castro-Camus, V. M. Palomar, and A. Covarrubias, "Leaf water dynamics of Arabidopsis thaliana monitored in-vivo using terahertz time-domain spectroscopy," Sci. Rep., vol. 3, Oct. 2013, Art. no. 2910

[9] R. Gente and M. Koch, "Monitoring leaf water content with $\mathrm{THz}$ and sub-THz waves," Plant Methods, vol. 11, no. 1, pp. 1-9, 2015

[10] L. G. Santesteban, I. Palacios, C. Miranda, J. C. Iriarte, J. B. Royo, and R. Gonzalo, "Terahertz time domain spectroscopy allows contactless monitoring of grapevine water status," Frontiers Plant Sci., vol. 6, p. 404, Jun. 2015.

[11] V. Torres et al., "Monitoring water status of grapevine by means of THz waves," J. Infr., Millim., THz. Waves, vol. 37, no. 5, pp. 507-513, 2016.

[12] J. Ma et al., "Estimating vegetation water content of corn and soybean using different polarization ratios based on L- and S-band radar data," IEEE Geosci. Remote Sens. Lett., vol. 14, no. 3, pp. 364-368, Mar. 2017.

[13] P. Ferrazzoli and L. Guerriero, "Passive microwave remote sensing of forests: A model investigation," IEEE Trans. Geosci. Remote Sens., vol. 34, no. 2, pp. 433-443, Mar. 1996.

[14] E. R. Hunt, L. Li, M. T. Yilmaz, and T. J. Jackson, "Comparison of vegetation water contents derived from shortwave-infrared and passivemicrowave sensors over central Iowa," Remote Sens. Environ., vol. 115, no. 9, pp. 2376-2383, 2011.

[15] R. Gente, A. Rehn, and M. Koch, "Contactless water status measurements on plants at $35 \mathrm{GHz}$," J. Infr., Millim., THz. Waves, vol. 36, no. 3 , pp. 312-317, Mar. 2015.

[16] D. M. Pozar, Microwave Engineering, 4th ed. Hoboken, NJ, USA: Wiley, 2012.

[17] K. Kupfer, Electromagnetic Aquametry: Electromagnetic Wave Interaction with Water and Moist Substances. Berlin, Germany: Springer, 2005.

[18] F. T. Ulaby, R. Moore, and A. Fung, Microwave Remote Sensing: Active \& Passive: From Theory to Applications, vol. 3. Norwood, MA, USA: Artech House, 1986.

[19] S. Ramo, J. R. Whinnery, and T. van Duzer, Fields and Waves in Communication Electronics, 3rd ed. New York, NY, USA: Wiley, 1994

[20] E. Baldini, Arboricultura General. Madrid, Spain: S.A. MUNDIPRENSA LIBROS, 1992

[21] L. Taiz and E. Zeiger, Plant Physiology and Development. Sunderland, MA, USA: Sinauer Associates, 2014

[22] J.-C. Fournioux and M. Adrian, Morphologie et Anatomie de la Vigne. Bordeaux, France: Féret, 2011.

[23] C. Pratt, "Vegetative anatomy of cultivated grapes-A review," Amer. $J$. Enol. Viticulture, vol. 25, no. 3, pp. 131-150, Jan. 1974.

[24] A. Jacobsen et al., "Grapevine xylem development, architecture, and function," in Functional and Ecological Xylem Anatomy. Springer, 2015, pp. $133-162$.

[25] K. Esau, "Phloem structure in the grapevine, and its seasonal changes," Hilgardia, vol. 18, no. 5, pp. 217-296, 1948.

[26] O. P. Rjabtschun, "Age-determined changes in the anatomic structure of the old axillary organs of vines," Mitt. Klosterneubg., vol. 22, no. 6, pp. 391-396, 1972.

[27] J.-W. Ting, D. Oloumi, and K. Rambabu, "FMCW SAR system for neardistance imaging applications-Practical considerations and calibrations," IEEE Trans. Microw. Theory Techn., vol. 66, no. 1, pp. 450-460, Jan. 2018

[28] A. A. Akram, D. Rozban, A. Abramovich, Y. Yitzhaky, and N. S. Kopeika, "Terahertz frequency modulated continuous wave radar using glow discharge detector," IEEE Sensors, vol. 16, no. 23, pp. 8440-8447, Dec. 2016.

[29] B. A. Atayants, V. M. Davydochkin, V. V Ezerskiy, V. S. Parshin, and S. M. Smolskiy, Precision FMCW Short-Range Radar for Industrial Applications. Norwood, MA, USA: Artech House, 2014.

[30] W. Liao, "MUSIC for multidimensional spectral estimation: Stability and super-resolution," IEEE Trans. Signal Process., vol. 63, no. 23, pp. 6395-6406, Dec. 2015.

[31] M. A. Abou-Khousa, D. L. Simms, S. Kharkovsky, and R. Zoughi, "High-resolution short-range wideband FMCW radar measurements based on MUSIC algorithm," in Proc. IEEE Instrum. Meas. Technol. Conf., May 2009, pp. 498-501.

[32] J. W. Odendaal, E. Barnard, and C. W. I. Pistorius, "Two-dimensional superresolution radar imaging using the MUSIC algorithm," IEEE Trans. Antennas Propag., vol. 42, no. 10, pp. 1386-1391, Oct. 1994.

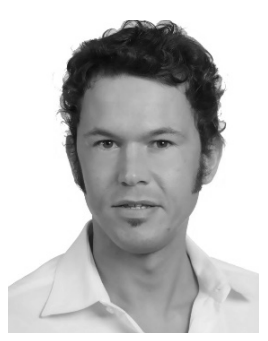

Carlos Quemada Mayoral received the B.Sc. and M.Sc. degrees in telecommunication engineering from the Public University of Navarre (UPNA), Pamplona, Spain, in 1999 and 2000, respectively, and the Ph.D. degree in RF integrated circuit (IC) design from the University of Navarra (TECNUN), San Sebastián, Spain, in 2006.

From 2008 to 2010, he was with the Technological Centre, IKERLAN, Mondragón, Spain, where he was involved in research and industrial projects on the design of embedded systems for RFID communications. In 2010, he joined the University of La Rioja, Logroño, Spain, as an Adjunct Professor. In 2011, he joined the Virginia Polytechnic Institute and State University, Blacksburg, VA, USA, as a Post-Doctoral Student of temperature compensated IC design. He is currently a Research Scientist with UPNA, where he is involved in the design, implementation, and measurement of frequency-modulated continuous-wave radars aimed at detecting biological fluids.

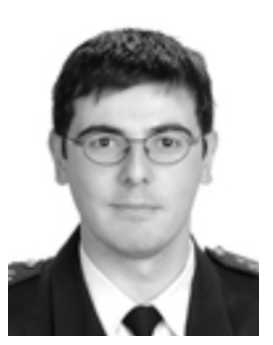

Cebrián García González was born in Pola de Laviana, Spain, in 1983. He received the M.Sc. and Ph.D. degrees in telecommunication engineering from the University of Oviedo, Gijón, Spain, in 2010 and 2016, respectively.

From 2010 to 2014, he was a Research Assistant of signal theory and communications area with the University of Oviedo, where he studied the inverse scattering problem. In 2013, he joined the ELEDIA Research Center, University of Trento, Trento, Italy, as a Visiting Student. From 2015 to 2017, he was a Research Assistant with the Antenna Group, Public University of Navarre, Pamplona, Spain, where he was involved in the development of a microwave radar for imaging applications. His research interests include the reconstruction of electromagnetic sources from field measurements, inverse scattering problem, and imaging techniques.

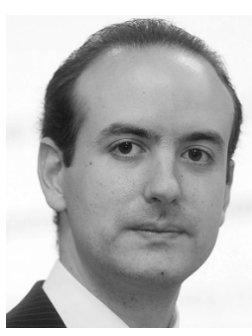

Juan Carlos Iriarte Galarregui was born in Pamplona, Spain, in 1978. He received the Ingeniero de Telecomunicación and Ph.D. degrees from the Public University of Navarre (UPNA), Pamplona, in 2002 and 2008, respectively.

Since 2001, he has been with the Antennas Group, Electrical and Electronic Engineering Department, UPNA. From 2002 to 2004, he was with, thanks to a University grant, the Antennas Group, where he was involved in the design of electromagnetic bandgap antennas. Since 2012, he has been an Associate Professor with UPNA. He has been involved in more than 67 research projects and contracts. He is the Founding Partner of the spin-off company Anteral S.L., Pamplona. His research interests include the THz imaging and sensing and metamaterials for microwave and millimeter-wave antenna applications with emphasis on space antenna applications.

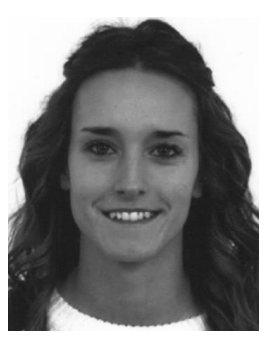

Diana Marín received the B.Sc. degree in agronomy and the M.Sc. degree in environmental agrobiology from the Public University of Navarre (UPNA), Pamplona, Spain, in 2015 and 2016, respectively, where she is currently pursuing the Ph.D. degree in biotechnology with the Advanced Fruit and Grape Growing Research Group, with a focus on the characterization of different factors that affect the quality of grapevine grafting plants.

From 2015 to 2017, she was a Project Assistant with the Advanced Fruit and Grape Growing Research Group, Department of Agronomy, Biotechnology and Food Science, UPNA, collaborating in different projects based on the agronomic evaluation in the field. She develops her research activity in collaboration with the Vitis Navarra nursery, which has developed a new line of vine rootstocks called RG series. Her research interests include the evaluation of the agronomic behavior of these RG series and the development of an objective method in order to measure the quality of the grafting plants. 


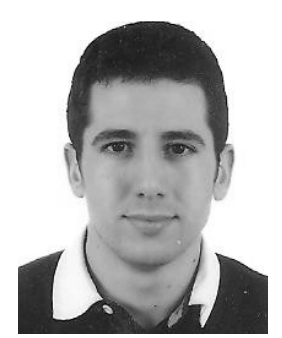

Diego Gastón received the B.Sc. degree in telecommunication technologies engineering and the M.Sc. degree in telecommunication engineering from the Public University of Navarre (UPNA), Pamplona, Spain, in 2015 and 2017, respectively.

Since 2014, he has been with Anteral S.L. Pamplona, a spin-off of the Antenna Group of UPNA. His research interests include the radar design and digital signal processing. He also searches for interesting applications of radar technology, where he develops custom software for his customers.

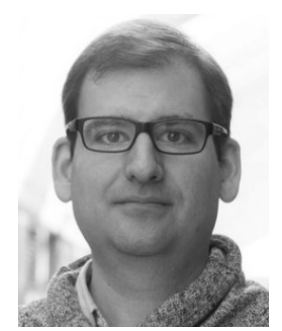

Carlos Miranda received the B.Sc. and Ph.D. (cum laude)degrees in agronomy from the Public University of Navarre (UPNA), Pamplona, Spain, in 1998 and 2003, respectively.

Since 1998, he has been a Researcher with the Advanced Fruit and Grape Growing Research Group, UPNA, where he is also an Associate Professor with the Department of Agronomy, Biotechnology and Food Science. He specializes in pomology and fruit tree genetic resources. His research interests include temperate fruit trees and grapevine, studies on the influence of climate, weather and crop management on productivity and fruit quality, precision agriculture and viticulture, and the evaluation of the genetic variability and characterization of wild and cultivated species. He has authored over 40 journal publications, more than 100 conference presentations, two books, and a number of technical documents and book chapters.

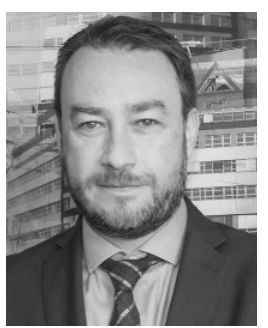

Ramón Gonzalo (M'94) received the M.Sc. and $\mathrm{Ph} . \mathrm{D}$. degrees (Hons.) in ingeniero de telecomunicación from the Public University of Navarre (UPNA), Pamplona, Spain, in 1995 and 2000, respectively.

Since 1995, he has been with the Antennas Group, Electrical and Electronic Engineering Department, UPNA, where he is currently a Full Professor. From 1997 to 1998, he was a Research Fellow of the Antenna Section, European Space Research and Technology Centre, European Space Agency, Noordwijk, The Netherlands. From 2008 to 2010, he was the Head of the Electrical and Electronic Engineering Department, UPNA, where he is a member of the Institute of Smart Cities. He has authored over 60 journal publications in peer-reviewed magazines and 100 conference papers related to his research lines.

Dr. Gonzalo was a co-recipient of the LAPC 2006, LAPC 2007, and IWAT 2007 Best Paper Award.

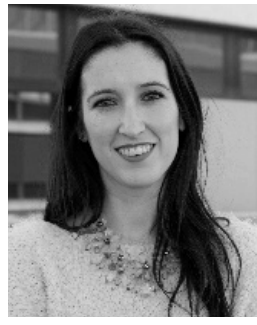

Itziar Maestrojuán received the B.S. degree in telecommunication engineering and the M.S. degree in communications from the Public University of Navarre (UPNA), Pamplona, Spain, in 2010 and 2011, respectively, and the Ph.D. degree (cum laude) in communications from UPNA, in 2015.

Since 2010, she has been a Ph.D. Student and a Junior Researcher with the Antenna Group, UPNA. She was a Visiting Research Scientist with RAL Space, Rutherford Appleton Laboratory, Harwell Oxford, Didcot, U.K., from 2011 to 2012. In 2014 she joined the Anteral S.L., Pamplona, as a THz Engineer, where she became the THz and Radar CTO in 2015 and the CEO in 2018. Her research interests include microwave and $\mathrm{THz}$ receivers design, imaging $\mathrm{THz}$ cameras, material characterization at $\mathrm{THz}$ range, and radar technology and innovative applications of it. She is a Contributor Author to the book Semiconductor THz Technology. She has authored several international journal papers and has attended more than 25 national and international conferences.

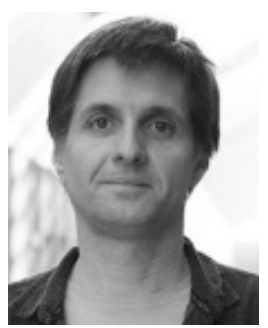

Luis Gonzaga Santesteban received the B.S. degree in agronomic engineering and the Ph.D. degree (cum laude) in agronomy and biotechnology from the Public University of Navarre (UPNA), Pamplona, Spain, in 1998 and 2003, respectively.

Since then, he has been with the Advanced Fruit and Grapevine Growing Research Team, where he has been the Leader since 2017. He is currently the Head of the Department of Agronomy, Biotechnology and Food Science, UPNA. His research interests include viticulture working on grapevine water relations, other aspects of grape growing such as canopy management and fertilization, precision viticulture, proximal sensing, and genetic diversity, side-to-side collaboration with wineries and winegrowers, combining the pursuit of new knowledge, and field implementation of current developments. He has authored more than 40 JCR indexed journal papers, given more than 50 conferences to grapegrowers and winemakers, and attended more than 50 national and international conferences.

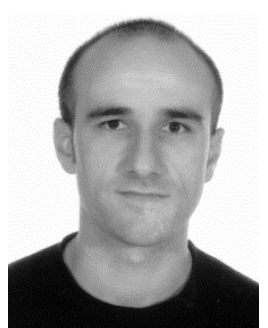

Iñigo Ederra (M'15) received the Ingeniero de Telecomunicación and Ph.D. degrees from the Public University of Navarre (UPNA), Pamplona, Spain, in 1996 and 2004, respectively.

In 1997, he joined the Microwave and Millimetre Wave Group, UPNA. From 1999 to 2000, he was with the European Space Research and Technology Centre, European Space Agency, Noordwijk, The Netherlands, where he was involved in electromagnetic bandgap materials and their applications in the field of antennas. Since 2001, he has been with the Antenna Group, UPNA. In 2002, he joined the Rutherford Appleton Laboratory, Chilton, Didcot, U.K., as Visitor Scientist, participating in ESA's Startiger I Project. He is currently an Associate Professor with UPNA and is a member of the Institute of Smart Cities. His research interests include electromagnetic bandgap materials, metamaterials, and metasurfaces and their applications in microwave, millimeter-wave, and $\mathrm{THz}$ components and antennas. 